\title{
Investigación del consumidor de aplicaciones móviles: hallazgos y perspectivas de estudio
}

Grupo de Investigación Mercadeo I+2

Oscar Robayo-Pinzon MSc. PhD @.

Julio de 2020

\section{Resumen}

El incremento en el uso del smartphone y las apps móviles ha generado para las marcas múltiples oportunidades de interacción con sus consumidores. En cuanto al tiempo de uso, se destacan las apps de redes sociales y mensajería instantánea, representando más del $50 \%$ del tiempo total de uso diario de los smartphones. Para abordar este fenómeno, la investigación del consumidor se ha enfocado principalmente en el proceso de adopción de estas soluciones móviles, destacándose la aplicación del modelo de aceptación de la tecnología. Por otra parte, se ha investigado la experiencia del usuario a través de la combinación de técnicas como el customer journey mapping y el eye tracking. Esto ha permitido explorar el proceso de compra de los usuarios en apps de comercio, principalmente en categorías de vestuario y calzado. Otro hallazgo relevante para las marcas consiste en las importantes diferencias que se han encontrado a nivel de usabilidad y valor percibido entre la página web y la app móvil de un mismo servicio. Se requiere entonces un minucioso estudio durante la fase de desarrollo de la app, para adaptar el contenido, las herramientas y la usabilidad de acuerdo al perfil y las expectativas del segmento de consumidores que se busca atraer. Finalmente, el estudio de la adopción, uso y valor percibido por parte de los consumidores con respecto a las apps móviles es un campo en pleno crecimiento, con desarrollos constantes en múltiples categorías de servicios.

Palabras clave: aplicaciones móviles, apps, customer journey mapping, eye tracking, modelo de aceptación de la tecnología, smartphone

\section{Abstract}

The increase in the use of smartphones and mobile apps has generated multiple opportunities for brands to interact with their consumers. In terms of usage time, social networking and instant messaging apps stand out, representing more than $50 \%$ of total daily smartphone usage time. To address this phenomenon, consumer research has focused mainly on the process of adoption of these mobile solutions, 
highlighting the application of the technology acceptance model. On the other hand, user experience research has been carried out through a combination of techniques such as customer journey mapping and eye tracking. This has allowed us to explore the purchase process of users in retail apps, mainly in clothing and footwear categories. Another relevant finding for the brands consists of the important differences found in the level of usability and perceived value between the website and the mobile app of the same service. A detailed study is then required during the development phase of the app, to adapt the content, tools and usability according to the profile and expectations of the consumer segment to be attracted. Finally, the study of adoption, use and perceived value by consumers with respect to mobile apps is a growing field, with constant developments in multiple service categories.

Key words: mobile apps, smartphone, customer journey mapping, eye tracking, technology acceptance model.

\section{Contexto actual del uso del smartphone y las apps}

El uso del teléfono inteligente ha aumentado considerablemente en los últimos años, permitiendo no sólo la comunicación inmediata con otras personas sino también facilitando muchas actividades diarias. Según el portal Statista (2020), se prevé que el número de usuarios de teléfonos inteligentes llegue a 3.500 millones en 2020, con una mayor tasa de penetración, que según Forrester Research (2018), superó la marca del $50 \%$ de la población mundial en 2017 , y se prevé que llegue al $66 \%$ en 2022. En el caso de América Latina, la tasa de penetración de la región será del $71 \%$ en 2020 , cifra superior a la tasa mundial del $66 \%$. Esto significa un total de 171 millones de usuarios adicionales para el final de la década (GSM Association, 2017). El aumento también es considerable desde el punto de vista del tiempo de uso. En los Estados Unidos, el tiempo medio de uso diario de los adultos fue de 3 horas y 35 minutos en 2018, lo que supone un aumento de 11 minutos con respecto al año anterior. Además, en 2019, el consumo de medios digitales móviles superó al de la televisión como el medio al que se dedican más minutos en los Estados Unidos (eMarketer Inc, 2019).

Dentro de este tiempo de uso de los dispositivos móviles, se destacan las aplicaciones móviles (Apps), las cuales representan más del $90 \%$ del tiempo total de actividad móvil (App Annie Intelligence, 2020). Aunque existen múltiples aplicaciones que han ido aumentando en número de descargas, en categorías como la mejora de la productividad, las comunicaciones en el trabajo, la autoayuda, la autopromoción y el entrenamiento físico, entre otras, son las categorías de redes sociales (social media) y las aplicaciones de mensajería 
instantánea las que siguen representando el $50 \%$ del tiempo total dedicado al uso de dispositivos móviles (Deloitte, 2019). Además, este tipo de aplicaciones fueron las que más contribuyeron al crecimiento del tiempo de uso de los teléfonos inteligentes durante los últimos años, por lo que se considera que hoy en día son una categoría con mayor madurez y hábitos más consolidados. Aun así, los usuarios a nivel mundial pasaron un $25 \%$ más de tiempo usando estas aplicaciones en 2019 en comparación con 2017 (App Annie Intelligence, 2020).Cabe destacar que dentro de las cinco aplicaciones con más usuarios activos a nivel mundial se encuentran cuatro del grupo de Facebook: WhatsApp Messenger, Facebook, Facebook Messenger, en los tres primeros lugares, e Instagram en el quinto lugar. En cuarto lugar, está We Chat, de la empresa china Tencent (Comscore, 2020).

\section{Búsqueda y descarga de las apps}

Dentro del estudio del comportamiento de los consumidores en el entorno de las apps móviles se ha considerado en primer lugar, cuáles pueden ser los factores que influyen en la búsqueda e intención de descarga de una app, lo cual resulta relevante en medio de la gran cantidad de opciones disponibles en las tiendas de apps de los sistemas operativos. De acuerdo con Statista (2020), existen 2,9 millones de apps disponibles en Google Play Store y 1,8 millones en el Apple App Store. Con respecto a la búsqueda de las apps en estas tiendas, se ha reportado que el $65 \%$ de las descargas provienen de búsquedas a través de palabras clave y el 35\% de las descargas se dan por un proceso de exploración no planeada de los usuarios en las App Stores (App Annie, 2017). Cuando se presenta el caso de una exploración no planeada, Lin \& Chen (2019) sugieren que dos elementos clave para facilitar la percepción y la consideración de una app son el ícono y la descripción, ya que estos son además, dos elementos que están dentro del ámbito de control del creador de la app. Estos autores manipularon diferentes condiciones del ícono y la descripción de una app ficticia para estudiar el efecto en la intención de descargar la app. Las condiciones involucraron dos opciones de diseño del ícono (estable/inestable) y dos tipos de descripciones (enfoque de promoción: búsqueda de logro, avance, cumplimiento de metas; y enfoque de prevención: protección, seguridad, prevención de riesgos). De forma interesante, sus resultados indican que los usuarios que se identificaron con un enfoque de promoción (prevención) tienen una mayor preferencia por un diseño de ícono inestable (estable). Es decir, que si se está buscando una percepción de confianza y seguridad en el potencial usuario, el diseño del ícono de la app debe ser más estable (simétrico), al tiempo que la descripción debe destacar la confiabilidad y seguridad en el manejo de los datos y la información del usuario.

Estudio de la experiencia de uso de las apps 
Otro importante aspecto de la experiencia de los usuarios con las apps, una vez se han convertido en usuarios frecuentes de la misma, es el recorrido o journey dentro de la interfaz, compuesta por menús, herramientas y pantallas, en las que se buscan diferentes objetivos, ya sea de compras, de servicios, de entretenimiento o de información. Una de las técnicas de mayor aplicación para entender la experiencia tal como la vive el consumidor es el customer journey mapping, la cual permite mejorar el diseño de la interfaz, el flujo de actividades y la optimización de los pasos dentro del proceso que debe llevar a cabo el usuario para obtener un resultado (Kuehnl, Jozic, \& Homburg, 2019). La técnica consiste en obtener un registro detallado de la experiencia del usuario con la app, para lo cual una de las maneras más efectivas de lograrlo es con el uso de la tecnología de eye-tracking. En un estudio reciente, la técnica se utilizó no para obtener los datos que normalmente se extraen de la medición, tales como fijaciones y patrones visuales, sino que por medio del registro en video de la experiencia desde la perspectiva del usuario a través de las diferentes pantallas de una app móvil, sumado a la técnica emergente conocida como retrospective think aloud o RTA por sus siglas en inglés, en la cual el usuario va narrando en voz alta lo que está percibiendo y sintiendo, a medida que va haciendo uso de la aplicación, con lo cual se pudo reconstruir paso a paso la experiencia completa de los usuarios. En resumen, los autores encontraron que al hacer uso de un sitio de retail de vestuario, el número promedio de pasos en la app móvil fue de 110, en tanto que en la versión web del mismo sitio, se obtuvieron apenas 66 pasos en promedio, con un tiempo promedio para todo el proceso de compra de un ítem, de entre $11 \mathrm{y}$ 12 minutos de duración (Tupikovskaja-Omovie \& Tyler, 2020).

\section{Estudios sobre la adopción de las apps}

También se debe considerar que la mayor parte de la literatura académica sobre comportamiento del consumidor en apps móviles corresponde al abordaje de la adopción de estas plataformas, especialmente en múltiples categorías de productos intangibles. Existen varios enfoques teóricos, entre ellos el desarrollado por (Sultan, Rohm, \& Gao, 2009), el cual propone un modelo conceptual que investiga la influencia de la actividad móvil asociada tanto al mercadeo como a la co-creación -que incluye el uso del móvil para acceder a la información y para compartir contenidos- en la aceptación que los consumidores tienen frente a las apps móviles de las marcas. Estos factores se derivan del Modelo de Aceptación de Tecnología (TAM) (Davis, 1989) y de la teoría de usos y gratificaciones, las cuales hacen hincapié en la influencia de los factores asociados con el uso, así como de las razones del uso en las intenciones conductuales. En esta perspectiva también se examina la influencia indirecta de dos factores antecedentes adicionales, el nivel de aceptación del riesgo percibido y el nivel de 
involucramiento o dependencia que tiene el usuario con su dispositivo móvil, en la aceptación de las apps móviles, particularmente en el segmento juvenil.

En consecuencia, el Modelo de Aceptación de Tecnología (TAM, por sus siglas en inglés) se basa en dos elementos que contribuyen a predecir la intención de adoptar el desarrollo tecnológico: la facilidad de uso percibida y la utilidad percibida (Davis, 1989). Aunque este modelo se ha aplicado principalmente en cuestiones relacionadas con la adopción de tecnología en las organizaciones, también se ha aplicado en contextos más generales relacionados con la adopción y el uso de diferentes innovaciones tecnológicas por parte de los consumidores (Davis, Bagozzi, \& Warshaw, 1989; Venkatesh, 2000). En el caso de la decisión de un consumidor de adoptar una app móvil, los motivadores incluyen la ventaja relativa que ofrece la app, el nivel de ajuste de la innovación ofrecida por las apps a las pautas de uso existentes, la complejidad percibida en el uso de la app, el riesgo percibido asociado al uso de la app, así como el nivel en el que el uso de la app es percibido y fomentado por otras personas (Özçam, Kusçu, \& Yozgat, 2015).

Además, el Modelo de Adopción de Tecnología (TAM) propuesto por Davis (1989), que a su vez se deriva de la Teoría de la Acción Razonada (TRA, por sus siglas en inglés) (Fishbein \& Ajzen, 1975), afirma que la utilidad percibida y la facilidad de uso percibida influyen no sólo en las actitudes hacia la innovación sino, finalmente, en su adopción. A diferencia de la TRA, el TAM permite que la utilidad percibida y la facilidad de uso desencadenen directamente la adopción de una app móvil, lo que refleja la noción de que no se requiere una actitud positiva para que la adopción tenga lugar. Por ejemplo, las normas de un grupo social pueden inducir muy directamente a los consumidores de ese grupo a adoptar una innovación (Venkatesh \& Davis, 2000). EI TAM está bien establecido como un modelo robusto y parsimonioso que predice la aceptación de una innovación tecnológica. Este modelo proporciona además una base para investigar el impacto de los factores externos en las creencias, actitudes e intenciones internas de utilizar productos tangibles e intangibles basados en la tecnología (Davis et al., 1989; Venkatesh, 2000).

Otro aspecto que ha resultado de gran interés para las empresas que buscan desarrollar su propia app móvil, consiste en las importantes diferencias que se han encontrado a nivel de usabilidad y valor percibido entre la página web y la app móvil de un mismo servicio. Los resultados de varios estudios señalan que se debe tener cuidado al tratar de extrapolar los resultados de las investigaciones sobre el uso y la percepción de los consumidores en los entornos tradicionales de Internet al entorno de uso de los dispositivos móviles. Por ejemplo, en un estudio sobre la adopción de servicios WAP (Protocolo de Aplicación Inalámbrica), se 
encontró que el nivel de utilización de los sitios de Internet móvil se reducía considerablemente debido a la aplicación incorrecta de los principios del diseño tradicional en entornos Web (Ramsay, 2000). Esto puede deberse en parte al hecho de que no se ha prestado suficiente atención al aspecto "móvil" del uso en diferentes tipos de actividades y procesos de intercambio de información en estos dispositivos (Shankar \& Balasubramanian, 2009).

\section{Conclusiones y perspectivas de estudio futuras}

Como se puede inferir de los anteriores casos y corrientes de investigación, el estudio de la adopción, uso y valor percibido por parte de los consumidores con respecto a las apps móviles es un campo en pleno crecimiento, con desarrollos constantes en múltiples categorías de servicios, por lo que hacia el futuro, el enfoque estará dado hacia las tendencias en consumo colaborativo y transformativo a nivel individual, familiar, social y ecológico, lo cual incluye el estudio de las apps que promuevan por ejemplo, comportamientos amigables con el medio ambiente, hábitos de alimentación saludables, el ahorro y la promoción de la actividad física, entre otros.

\section{Referencias}

App Annie. (2017). 2017 Retrospective: A Monumental Year for the App Economy. Retrieved July 1, 2020, from https://www.appannie.com/en/insights/marketdata/app-annie-2017-retrospective/\#: :text=Today\%2C we're excited to,from the top regional markets

App Annie Intelligence. (2020). State of Mobile 2020. Retrieved from https://www.appannie.com/en/go/state-of-mobile-2020/

Comscore. (2020). Global State of Mobile. Retrieved from https://www.comscore.com/Insights/Presentations-andWhitepapers/2019/Global-State-of-Mobile

Davis, F. D. (1989). Perceived usefulness, perceived ease of use, and user acceptance of information technology. MIS Quarterly, 13(3), 319-339.

Davis, F. D., Bagozzi, R. P., \& Warshaw, P. R. (1989). User acceptance of computer technology: a comparison of two theoretical models. Management Science, 35(8), 982-1003.

Deloitte. (2019). Consumo móvil en Colombia. Bogotá. Retrieved from https://www2.deloitte.com/content/dam/Deloitte/co/Documents/consumerbusiness/Reporte consumo movil 2019.pdf

eMarketer Inc. (2019). Average Time Spent with Media in 2019 Has Plateaued. Retrieved June 18, 2020, from https://www.emarketer.com/content/us-timespent-with-media-in-2019-has-plateaued-with-digital-making-up-losses-by-old- 
media

Fishbein, M., \& Ajzen, I. (1975). Belief, Attitude, Intention, and Behavior: An Introduction to Theory and Research. Reading, MA: Addison-Wesley.

Forrester Research. (2018). Forrester Data: Mobile, Smartphone, And Tablet Forecast, 2017 To 2022. Retrieved from https://www.forrester.com/report/Forrester+Data+Mobile+Smartphone+And+T ablet+Forecast+2017+To+2022+Global/-/E-RES138971

GSM Association. (2017). The Mobile Economy Latin America and the Caribbean 2017. Retrieved from https://www.gsma.com/latinamerica/mobile-economylatin-america-caribbean-2017/

Kuehnl, C., Jozic, D., \& Homburg, C. (2019). Effective customer journey design: consumers' conception, measurement, and consequences. Journal of the Academy of Marketing Science, 47(3), 551-568. https://doi.org/10.1007/s11747-018-00625-7

Lin, C.-H., \& Chen, M. (2019). The icon matters: how design instability affects download intention of mobile apps under prevention and promotion motivations. Electronic Commerce Research, 19(1), 211-229. https://doi.org/10.1007/s10660-018-9297-8

Özçam, D. S., Kusçu, A., \& Yozgat, U. (2015). Understanding mobile advertising acceptance: An integrative approach. International Journal of Mobile Communications, 13(4), 376-397. https://doi.org/10.1504/IJMC.2015.070061

Ramsay, M. (2000). WAP usability report.

Shankar, V., \& Balasubramanian, S. (2009). Mobile Marketing: A Synthesis and Prognosis. Journal of Interactive Marketing, 23(2), 118-129. https://doi.org/10.1016/j.intmar.2009.02.002

Statista. (2020). Number of smartphone users worldwide from 2016 to 2021. Retrieved July 13, 2020, from https://www.statista.com/statistics/330695/number-of-smartphone-usersworldwide/

Sultan, F., Rohm, A. J., \& Gao, T. (T. . (2009). Factors Influencing Consumer Acceptance of Mobile Marketing: A Two-Country Study of Youth Markets. Journal of Interactive Marketing, 23(4), 308-320. https://doi.org/10.1016/j.intmar.2009.07.003

Tupikovskaja-Omovie, Z., \& Tyler, D. (2020). Clustering consumers' shopping journeys: eye tracking fashion m-retail. Journal of Fashion Marketing and Management. https://doi.org/10.1108/JFMM-09-2019-0195

Venkatesh, V. (2000). Determinants of Perceived Ease of Use: Integrating Control, 
Intrinsic Motivation, and Emotion into the Technology Acceptance Model. Information Systems Research, 11(4), 342-365. https://doi.org/10.1287/isre.11.4.342.11872

Venkatesh, V., \& Davis, F. D. (2000). A theoretical extension of the technology acceptance model: four longitudinal field studies. Management Science, 46(2), 186-204. 